\title{
Erratum: Prediction for a Four-Neutron Resonance [Phys. Rev. Lett. 117, 182502 (2016)]
}

\author{
A. M. Shirokov, G. Papadimitriou, A. I. Mazur, I. A. Mazur, R. Roth, and J. P. Vary
}

(Q) (Received 13 August 2018; published 31 August 2018)

DOI: 10.1103/PhysRevLett.121.099901

The minus sign is missed in the right-hand side of Eq. (7) of this Letter; the corrected Eq. (7) is

$$
\phi(E)=-\frac{w_{1} \sqrt{E}+w_{3}(\sqrt{E})^{3}+c(\sqrt{E})^{5}}{1+w_{2} E+w_{4} E^{2}+w_{6} E^{3}+d E^{4}} .
$$

There are also misprints in the values of rms deviations of energies and in the units of the fit parameter $a$. The third line in the left column of page 182502-4 below Fig. 2 should read

$$
\text { "rms deviation of } 41 \mathrm{keV} \text { with } a=0.724 \mathrm{MeV}^{1 / 2} \text {," }
$$

and the fourth and fifth lines below Eq. (9) in the right column of page 182502-4 should read

$$
\text { "rms deviation of } 44 \mathrm{keV} \text { with the parameters } a=0.701 \mathrm{MeV}^{1 / 2}, b^{2}=1.089 \mathrm{MeV}, c=-27.0 \mathrm{MeV}^{-5 / 2} . "
$$

The results and conclusions of this Letter are not affected. 